\title{
ROTAÇÃO DE RETALHO PALATINO COMO ALTERNATIVA DE TRATAMENTO PARA FÍSTULA BUCOSSINUSAL
}

\section{PALATINE FLAP ROTATION AS AN ALTERNATIVE TREATMENT FOR OROANTRAL FISTULA}

\section{Eugênio Rodrigues Arantes}

Residente de Cirurgia Oral e Maxilofacial do Hospital Federal dos Servidores do Estado do Rio de Janeiro - HFSE e da Universidade Federal Fluminense - UFF.

Rafael Mérola Corrêa

Residente de Cirurgia Oral e Maxilofacial do Hospital Federal dos Servidores do Estado do Rio de Janeiro - HFSE e da Universidade Federal Fluminense - UFF.

Suelen Cristina Sartoretto

Professora das disciplinas de Cirurgia Bucal, Semiologia e Anatomia da Faculdade de Odontologia da Universidade Iguaçu - UNIG.

Rodrigo Figueiredo

Professor da disciplina de Cirurgia Bucal da Universidade Federal Fluminense - UFF e Professor das disciplinas de Cirurgia Bucal, Semiologia e Clínica Integrada da Faculdade de Odontologia da Universidade Iguaçu - UNIG.

Instituição na qual o trabalho foi realizado: Hospital Federal dos Servidores do Estado do Rio de Janeiro/RJ

Categoria: Relato de caso

\section{Informações do autor principal:}

Eugênio Braz Rodrigues Arantes

Rua Mário Santos Braga, 28

Centro - Niterói - RJ, Brasil.

CEP: $24020-140$

E-mail: ebrarantes@gmail.com

Telefone: +552126299920 


\title{
Resumo
}

A fístula ou comunicação bucossinusal consiste na formação de um trajeto direto entre a cavidade oral e o seio maxilar diagnosticada tardiamente após epitelização da mucosa formando um canal bucossinusal permanente. Na maioria das vezes, essa complicação ocorre em função da relação anatômica do seio maxilar intimamente relacionado com o ápice das raízes dos dentes superiores posteriores. Procedimentos cirúrgicos inadequados relacionados à exodontia dos elementos dentários envolvidos ou extensa pneumatização do seio maxilar podem ser as causas mais comuns dessa patologia. $\mathrm{O}$ objetivo do presente trabalho é apresentar um relato de caso clínico de uma fístula bucossinusal pós exodontia e não corrigida imediatamente, tratada através de retalho mucoso palatino vascularizado e rotacionado para fechamento primário. A técnica do retalho palatino mostrou-se uma opção favorável para o fechamento do defeito sinusal em um único tempo cirúrgico, preservando a mucosa queratinizada e a anatomia do sulco vestibular.

Palavras-chave: Seio maxilar, sinusite maxilar, fístula bucoantral, cirurgia bucal.

\begin{abstract}
The bucosinusal fistula or communication consists in the formation of a direct path between the oral cavity and the maxillary sinus diagnosed late after epithelialization of the mucosa forming a permanent bucosinusal canal. Most often, this complication occurs due to the anatomical relationship of the maxillary sinus closely related to the apex of the roots of the posterior superior teeth. Inadequate surgical procedures related to the extraction of the involved dental elements or extensive pneumatization of the maxillary sinus may be the most common causes of this pathology. The aim of the present study is to present a case report of an immediately uncorrected post-extraction bucosinusal fistula treated with a vascularized palatine mucous flap and rotated for primary closure. The palatal flap technique proved to be a favorable option for closing the sinus defect in a single surgical time, preserving the keratinized mucosa and the buccal sulcus anatomy.
\end{abstract}

Key-words: Maxillary sinus, maxillary sinusitis, oroantral fistula, oral surgery. 


\section{Introdução}

O trajeto direto entre o do seio maxilar e a cavidade oral é conhecido como fístula ou comunicação bucossinusal. Os seios maxilares, localizados bilateralmente à cavidade nasal, quando em sua função e forma normais, são revestidos por um epitélio diferenciado, pseudoestratificado cilíndrico ciliado, de grande importância para a sua fisiologia e que contribui para a drenagem e secreção de muco como coadjuvantes na função respiratória (PETERSON LJ, ELLIS E, ET AL. 1988). Em situações de comunicação, quando o diagnóstico é feito tardiamente, este caminho pode sofrer epitelização permanente, ou seja, passa a ser revestido por tecido epitelial, configurando uma fístula bucossinusal (PETERSON LJ, ELLIS E, ET AL. 1988; EPPLEY B, SCAROFF A. 1984).

A fisiologia e anatomia dos seios maxilares podem favorecer o aparecimento de complicações bucossinusais, assim como, a extração de dentes superiores posteriores e a uma grande pneumatização da mucosa sinusal. A proximidade de dentes superiores posteriores com raízes longas ou com grandes dilacerações e a grande pneumatização do seio maxilar associada à fragilidade capilar da mucosa sinusal são os principais fatores para o surgimento desta condição que pode surgir acidentalmente durante o procedimento cirúrgico de extração dentária (ARAÚJO A, GABRIELLI MFR, ET AL. 2007; DEMETOGLU U, OCAK H, ET AL. 2018).

O diagnóstico da fístula bucossinusal interfere diretamente na escolha do método de tratamento para esta patologia. Seus sinais e sintomas variam de acordo com o tamanho e destruição local causada pela manipulação cirúrgica. A passagem de líquidos entre as cavidades oral e nasal, voz anasalada, sinusite maxilar recorrente e a disosmia são sintomas mais comuns, e, como método de diagnóstico coadjuvante, a manobra de Valsalva pode ser utilizada para confirmar a presença ou não da comunicação clinicamente (ARAÚJO A, GABRIELLI MFR, ET AL. 2007). Exames radiográficos convencionais podem ser utilizados como métodos coadjuvantes, eles auxiliam na identificação de um defeito ósseo na região e um espessamento da mucosa sinusal (KAPUSTECKI M, NIEDZIELSKA I, ET AL. 2016). 
As complicações bucossinusais que acontecem acidentalmente durante o ato cirúrgico de extrações dentárias, limitadas a região apical, tendem a evoluir para a regressão espontânea do caso, desde que não haja sinusite maxilar prévia (ARAÚJO A, GABRIELLI MFR, ET AL. 2007; MITCHELL R, LAMB J. 1983). A localização, extensão, grau de acometimento do seio maxilar e a presença ou não de infecção associada poderão interferir no tratamento final, que vai desde o fechamento primário do alvéolo até os retalhos cirúrgicos utilizando enxertos autógenos ou materiais aloplásticos (MITCHELL R, LAMB J. 1983).

Dessa forma, o objetivo deste trabalho é o retalho palatino mucoso como alternativa de tratamento para um paciente portador de fístula bucossinusal pós exodontia.

\section{Considerações éticas}

Os procedimentos empregados neste trabalho seguiram os padrões éticos propostos pela Declaração de Helsinque de 1975, revisada em 2000. O paciente foi tratado no Hospital Federal dos Servidores do Estado do Rio de Janeiro conveniado com a Universidade Federal Fluminense através da Residência em Cirurgia Oral e Maxilofacial com os termos, de consentimento livre e esclarecido e de uso de imagens e dados para publicação científica, assinados.

O estudo foi escrito seguindo as Diretrizes do Relatório de Caso Clínico (CARE, www.care-statement.org).

\section{Relato de caso}

Paciente do sexo masculino, 59 anos de idade, edêntulo total superior, procurou o Serviço de Cirurgia Oral e Maxilofacial do Hospital Federal dos Servidores do Estado do Rio de Janeiro após realizar extração dentária há aproximadamente 2 anos. Durante o exame clínico, o paciente queixou-se de dor em hemiface esquerda, "gosto ruim na boca" e presença de um orifício na gengiva. Ao exame intraoral, observou-se uma fístula em rebordo alveolar superior de aproximadamente $1 \mathrm{~cm}$ na região correspondente ao primeiro molar superior esquerdo extraído previamente (Figura 1A). 


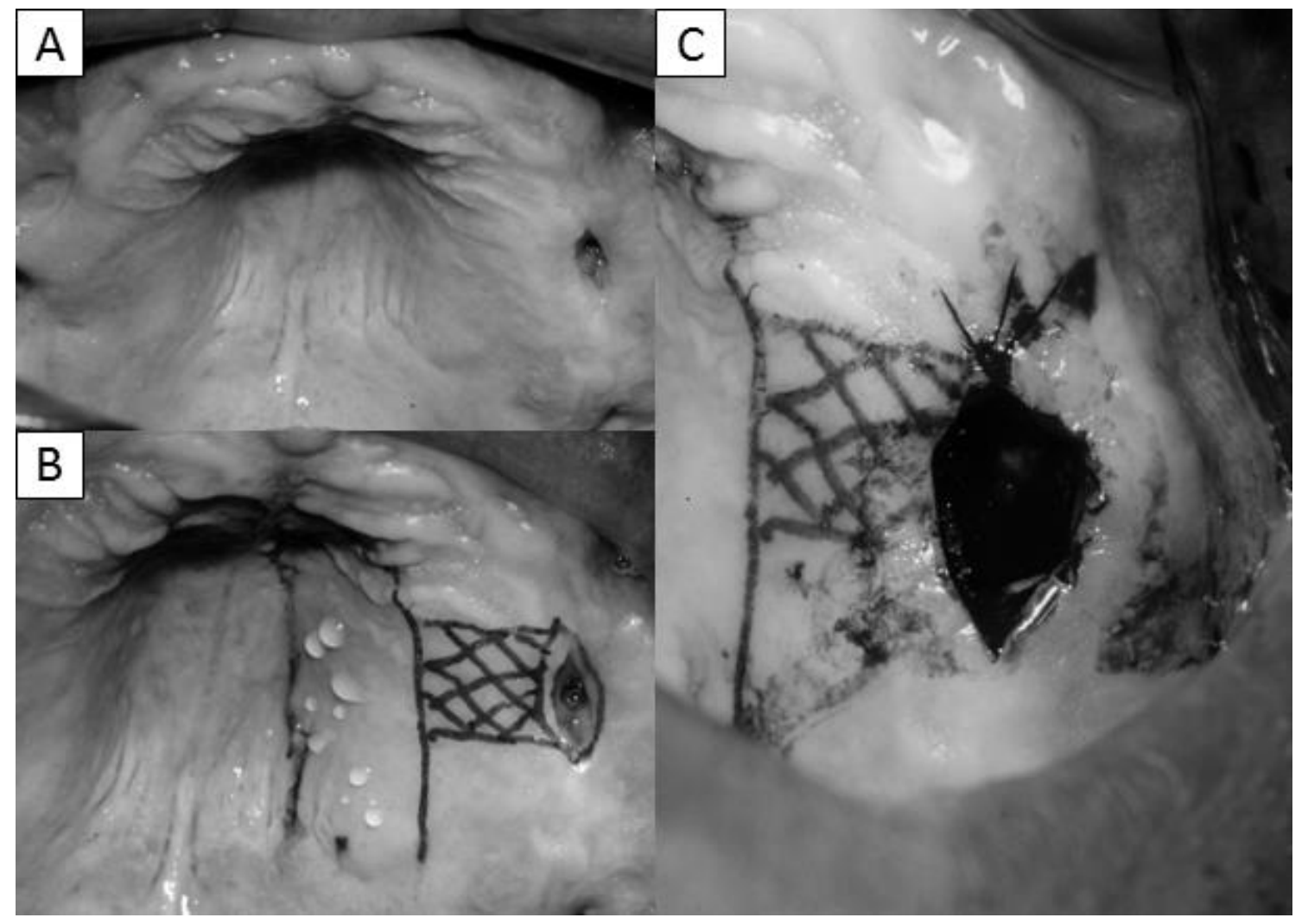

Figura 1. A) Imagem inicial evidenciando fistula de aproximadamente $1 \mathrm{~cm}$ de diâmetro na área de molares superiores. B) Marcação do retalho que será reposicionado. C) Área da fístula ressecada.

O Teste de Valsalva foi realizado para confirmar a presença da comunicação entre a cavidade oral e a cavidade sinusal e, após a manobra, pôde-se observar também uma drenagem purulenta através do orifício, confirmando a presença de uma fístula bucossinusal crônica. Previamente a manipulação cirúrgica, o paciente foi orientado a realizar inalação com Penetro ${ }^{\circledR}$ (Eucaliptol 33mg/ml + Terpina Monoidratada $22 \mathrm{mg} / \mathrm{ml}$ + Mentol 22mg/ml - Laboratório Daudt, Rio de Janeiro, BR) associada a irrigação com Soro Fisiológico $0,9 \%{ }^{\circledR}$ (Cloreto de sódio 0,9g - Eurofarma, São Paulo, BR) duas vezes ao dia. Além disso, foi realizada antibioticoterapia com Clavulin® BD (Amoxicilina 875mg + Clavulanato de potássio 125mg - GlaxoSmithKline Brasil Ltda., Rio de Janeiro, BR) durante os 7 dias que antecederam a cirurgia. Após remissão do quadro de drenagem local, a paciente foi submetida ao procedimento cirúrgico.

O procedimento cirúrgico foi realizado a nível ambulatorial e sob anestesia local através de ressecção do epitélio da fístula localizado no rebordo alveolar (Figuras 1 B e 
C). Em seguida foi conduzida a sinusectomia maxilar para remoção da membrana infectada e de todo o trato fistuloso. O retalho palatino pediculado ipsilateral à fístula medindo aproximadamente $4 \mathrm{~cm}$ foi realizado de forma vascularizada através da conservação da artéria palatina e girado com a intenção de cobrir a comunicação preexistente (Figura 2). A ferida cirúrgica foi fechada em primeira intenção através de suturas $\left(\right.$ Ethicon $^{\circledR}$ - Johnson \& Johnson Medical, New Brunswick, USA) e o sítio doador composto pela área de palato duro manteve-se cruenta para fechamento em segunda intenção.

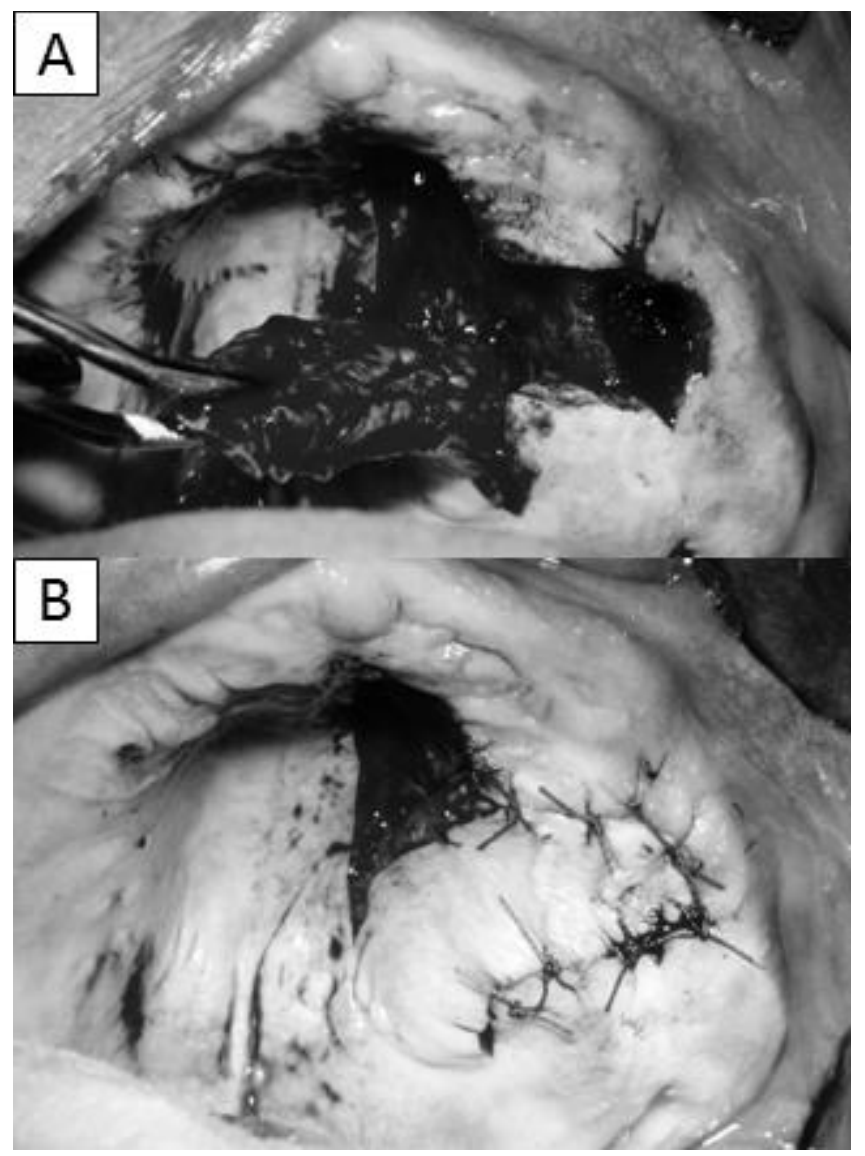

Figura 2. A) Retalho mucoperiosteo total de palato e área da fistula ressecada. B) Retalho palatino vascularizado rodado para fechamento da fistula com a síntese tecidual realizada.

Ao final do procedimento cirúrgico pôde-se perceber boa aproximação das bordas da ferida e ausência de tensão no retalho palatino. Foi produzido um dispositivo, em resina acrílica, para proteção do leito palatino doador e da ferida cirúrgica, associado ao curativo com cimento cirúrgico TechNew ${ }^{\circledR}$ (Composição pó: Acetato de zinco, ácido 
tânico, óxido de zinco e celulose + líquido: eugenol, óleo de oliva e corante EUROTECHNEW Com. Imp. Exp. de Materiais Dentários Ltda., Canelas, PT) durante 15 dias de pós-operatório inicial até ocorrer a granulação tecidual da área operada (Figura 3A). O uso de Clavulin® BD (Amoxicilina 875mg + Clavulanato de potássio 125mg - GlaxoSmithKline Brasil Ltda., Rio de Janeiro, BR) se manteve por sete dias após o procedimento cirúrgico. No pós-operatório imediato, o paciente apresentou boa evolução, sem complicações na ferida cirúrgica e com resolução das queixas prévias. Em acompanhamento de um ano, ao exame intraoral e de imagem foi possível observar boa cicatrização dos tecidos moles e ausência de fístula bucossinusal (Figura 3B).

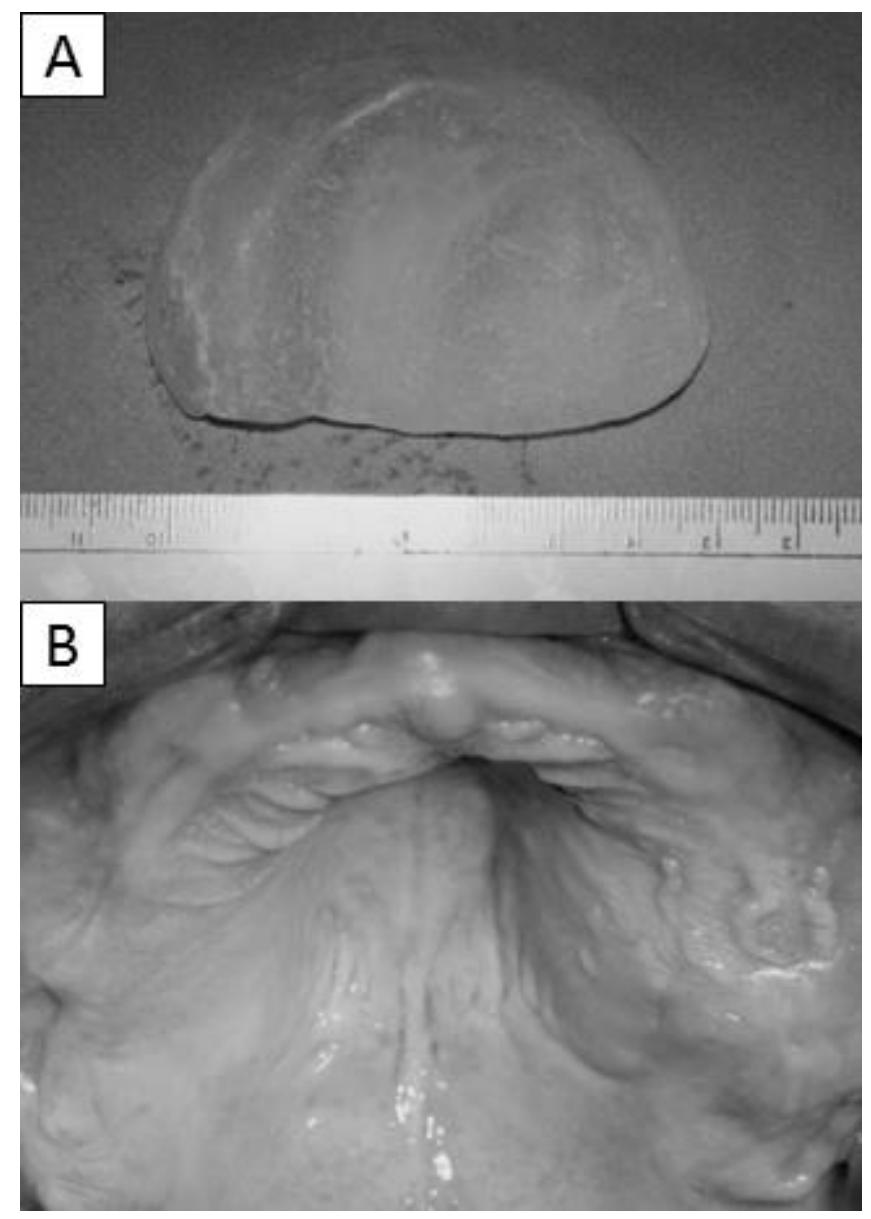

Figura 3. A) Placa de acrílico confeccionada para auxiliar o processo de cicatrização pós-operatória. B) Pós-operatório de 12 meses evidenciando boa cicatrização do retalho e fechamento da fístula.

\section{Discussão}


O tamanho e extensão da comunicação bucossinusal e consequentemente da fístula gerada por esta complicação irão nortear a estratégia de tratamento a ser escolhida para cada caso. Em suma, a complexidade da manipulação cirúrgica é diretamente proporcional a extensão da fístula bucossinusal, exigindo a realização de retalhos cirúrgicos específicos com ou sem a associação de outras técnicas auxiliares (ARAÚJO A, GABRIELLI MFR, ET AL. 2007). Defeitos de pequenas proporções causados acidentalmente durante extrações dentárias, não demonstram grandes dificuldades no seu fechamento e podem ser tratados através do fechamento primário do alvéolo para manutenção do coágulo e formação de tecido cicatricial (ARAÚJO A, GABRIELLI MFR, ET AL. 2007; RAPIDIS AD, ALEXANDRIDIS CA, ET AL. 2000; DE SOUZA LOPES PH, SAMPAIO DO, ET AL. 2015).

As comunicações bucossinusais consideradas imediatas, livres de processo infeccioso, quando acidentais e de pequena magnitude (aproximadamente 1-3 mm de diâmetro), geralmente, evoluem com bons resultados, quando o fechamento primário é realizado com aproximação das bordas da gengiva, sem tensão com ou sem alveoloplastias das cristas ósseas (ARAÚJO A, GABRIELLI MFR, ET AL. 2007; DE SOUZA LOPES PH, SAMPAIO DO, ET AL. 2015). Por estarem associadas ao momento da extração dentária, geralmente são limitadas a região apical e sua evolução acompanha o processo de cicatrização alveolar (RAPIDIS AD, ALEXANDRIDIS CA, ET AL. 2000; DE SOUZA LOPES PH, SAMPAIO DO, ET AL. 2015). Uma comunicação bucossinusal imediata mal conduzida pode provocar a infecção do seio maxilar e, a cronicidade desse evento, cursando induz a formação de fístulas necessitando de um procedimento cirúrgico secundário para o seu fechamento (ARAÚJO A, GABRIELLI MFR, ET AL. 2007).

Na presença de um processo infeccioso sinusal, acompanhado de sintomatologia dolorosa, velamento radiográfico do seio maxilar e drenagem de pus, o tratamento da infecção é realizado previamente ao tratamento cirúrgico. O antibiótico de primeira escolha para o tratamento deste tipo infecção é o grupo das Penicilinas, como representante a Amoxicilina, prescrita no pré-cirúrgico com a função de debelar a infecção e preparar o seio maxilar para a manipulação cirúrgica (DE SOUZA LOPES PH, SAMPAIO DO, ET AL. 2015). Como protocolo de tratamento das sinusites maxilares, inalantes, descongestionantes nasais e irrigação do seio maxilar devem ser REVISTA FLUMINENSE DE ODONTOLOGIA - ANO XXVI - No 53 - Janeiro / Julho 2020 
adicionados com o objetivo de diminuir o edema da mucosa sinusal, facilitar a drenagem das secreções e impedir a obliteração do óstio. Após estabilização do processo infeccioso e melhora na sintomatologia sinusal, o tratamento cirúrgico pode ser planejado (KAPUSTECKI M, NIEDZIELSKA I, ET AL. 2016; RAPIDIS AD, ALEXANDRIDIS CA, ET AL. 2000; DE SOUZA LOPES PH, SAMPAIO DO, ET AL. 2015).

Várias técnicas cirúrgicas são indicadas para o fechamento das comunicações, tais como: rotações locais (retalhos vestibulares e palatinos), retalhos à distância como músculo temporal e língua, o uso de enxerto bucal pediculado de gordura (bola de Bichat), a utilização de enxertos ósseos autógenos e de materiais aloplásticos (ARAÚJO A, GABRIELLI MFR, ET AL. 2007; MITCHELL R, LAMB J. 1983). Diversos retalhos cirúrgicos podem ser realizados para o fechamento de fístulas bucossinusais, principalmente nos casos em que um maior relaxamento dos tecidos é necessário ou quando uma grande quantidade de tecido deve ser obtida para a correção do defeito (RAPIDIS AD, ALEXANDRIDIS CA, ET AL. 2000). Para a realização dos retalhos cirúrgicos, é imprescindível o avivamento das margens da ferida associado à ressecção da fístula (fistulectomia), tornando-a cruenta e capaz de cicatrizar com o retalho (PETERSON LJ, ELLIS E, ET AL. 1988; AD, ALEXANDRIDIS CA, ET AL. 2000).

No caso clínico descrito, a escolha do retalho palatino foi baseada no tamanho e localização da fístula, na ausência da infecção sinusal e na disponibilidade de tecidos moles uma vez que os retalhos vestibulares impossibilitariam a confecção de uma nova prótese posteriormente. Uma série de vantagens está relacionada à utilização do retalho palatino para o fechamento de fístulas bucossinusais como: (1) a presença de uma mucosa espessa facilitando o fechamento e cobertura do defeito, (2) a manutenção do fundo de sulco vestibular e (3) a melhor vascularização preservada pelo aporte sanguíneo da artéria palatina presente em seu interior (PETERSON LJ, ELLIS E, ET AL. 1988; ARAÚJO A, GABRIELLI MFR, ET AL. 2007). Das limitações presentes nessa técnica, a dificuldade para posicionamento e imobilização do retalho palatino como a possibilidade de necrose tecidual, hemorragia acidental da artéria palatina e o desconforto gerado devido a área doadora cruenta, são os mais descritos na literatura (ARAÚJO A, GABRIELLI MFR, ET AL. 2007; KAPUSTECKI M, NIEDZIELSKA I, ET AL. 2016).

REVISTA FLUMINENSE DE ODONTOLOGIA - ANO XXVI - No 53 - Janeiro / Julho 2020 
Como descrito anteriormente, o retalho palatino é ricamente suprido pela artéria palatina maior e por inúmeros vasos de menor calibre localizados por todo o palato. Além disso, a espessura, largura e textura da região palatina favorecem a manipulação deste retalho, permitindo a rotação do mesmo (ARAÚJO A, GABRIELLI MFR, ET AL. 2007; KAPUSTECKI M, NIEDZIELSKA I, ET AL. 2016). Os autores deste caso clínico optaram por utilizar o retalho palatino como manobra cirúrgica devido aos diversos fatores vantajosos descritos, como a excelente nutrição combinada com espessura e textura do retalho. A utilização do retalho mucoso rotacionado do palato se mostrou previsível com um fechamento bem sucedido da fístula, bem como a reepitelização da área doadora. Esta técnica permitiu uma cicatrização ideal com uma faixa abundante de mucosa queratinizada.

\section{Conclusão}

Diversas formas de tratamento estão descritas na literatura para o fechamento de comunicações e fístulas bucossinusais, no entanto, os mecanismos de prevenção de complicações são as melhores formas para evitar que elas ocorram. No caso clínico realizado, a utilização do retalho rotatório palatino demonstrou-se adequada e previsível durante o período de acompanhamento de um ano com excelente cicatrização tecidual e eliminação total dos sinais e sintomas sinusais.

\section{Referências}

1. Peterson LJ, Ellis E, et al. Contemporary oral and maxillofacial surgery. 3rd Ed. St. Louis: Mosby; 1988.

2. Eppley B, Scaroff A. Oro-nasal fistula secondary to maxillary augmentation. Int $\mathbf{J}$ Oral Surg. 1984;13(6):535-8.

3. Araújo A, Gabrielli MFR, et al. Aspectos atuais da cirurgia e traumatologia bucomaxilofacial. 1st Ed. Editora Santos; 2007.

4. Demetoglu U, Ocak H, et al. Closure of oroantral communication with plasma-rich fibrin membrane. J Craniofacial Surg. 2018; 29(4):367-370.

5. Kapustecki M, Niedzielska I, et al. Alternative method to treat oroantral communication and fistula with autogenous bone graft and platelet rich fibrin. Med Oral Patol Oral Cir Bucal. 2016; 21(5):608-613. 
6. Mitchell R, Lamb J. Immediate closure of oro-antral communications with a collagen implant. A preliminary report. Br Dent J. 1983; 19-154(6):171-4.

7. Rapidis AD, Alexandridis CA, et al. The use of the buccal fat pad for reconstruction of oral defects: review of the literature and report of 15 cases. J Oral Maxillofac Surg. 2000; 58:158-63.

8. de Souza Lopes PH, Sampaio DO, et al. Combined palatal flap and titanium mesh for oroantral fistula closure. Ann Maxillofac Surg. 2015;5(1):89-92. 